Pacific

Journal of

Mathematics

\title{
GENERIC BEHAVIOR OF ITERATED FUNCTION SYSTEMS WITH OVERLAPS
}

Michat RAMS 


\title{
GENERIC BEHAVIOR OF ITERATED FUNCTION SYSTEMS WITH OVERLAPS
}

\author{
MICHAŁ RAMS
}

\begin{abstract}
We prove that for typical self-similar iterated function systems satisfying the transversality condition the number of intersections of cylinders of a given size is for many sizes nearly as small as possible. This implies a result of Solomyak on the absolute continuity of the natural measure and a result of Peres, Simon and Solomyak on the positivity of the packing measure.
\end{abstract}

\section{Introduction}

Let $V$ be an open and bounded subset of $\mathbb{R}^{d}$. For each parameter value $t \in \bar{V}$ we consider a linear conformal iterated function system (IFS) $\left(f_{i}\right)_{i=1}^{k}$ in $\mathbb{R}^{d}$ depending on $t$. We assume the dependence on $t$ is differentiable, to class at least $C^{1+\beta}$. We denote by $\Lambda_{t}$ the limit set of the IFS attached to a given value of $t$, and by $s(t)$ the solution of the Moran equation

$$
\sum \lambda_{i}^{s}=1
$$

where $\lambda_{i}$ is the contraction ratio of $f_{i}$. If the IFS satisfies the open set condition (OSC), it is a classical result [Hutchinson 1981] that limit set $\Lambda_{t}$ has Hausdorff dimension $s(t)$. If the OSC is not satisfied, the Hausdorff dimension of $\Lambda_{t}$ may be smaller than $s(t)$.

The natural measure $v$ is the unique probability measure satisfying the equation

$$
v=\sum \lambda_{i}^{s} \nu \circ f_{i}^{-1}
$$

If the OSC is satisfied, this measure is equivalent to the $s$-dimensional Hausdorff measure (or the packing measure - they are equivalent in this case) on $\Lambda_{t}$. In this paper we study the properties of $v$ and $\Lambda$ without assuming the OSC.

In [Rams 2002] we introduced intersection numbers and proved some bounds for them. Here we prove stronger bounds (Theorem 4.5), under stronger assumptions, and use them to give new proofs of two theorems. The first is Theorem 5.4, which states that for almost all parameter values for which $s(t)>d$ the natural measure is equivalent to the $d$-dimensional Lebesgue measure on $\Lambda_{t}$. A statement of this

MSC2000: 37C45.

This research was supported by Polish KBN Grant No. 2 P03A 009 17, PRODYN and Foundation for Polish Science. 
kind was first proved in [Solomyak 1995]; the more general version may be found in [Peres and Schlag 2000]. The second result, Theorem 5.6, states that for almost all parameter values for which $s(t)<d$ the packing measure of $\Lambda_{t}$ is positive and equivalent to the natural measure. This was first proved in [Peres et al. 2000].

Section 2 provides background and notation. In Section 3 we introduce the transversality condition and quote a few lemmas proved in [Rams 2002]. Section 4 contains the proof of our main result, Theorem 4.5. From that we derive Theorems 5.4 and 5.6 as easy consequences in Section 5.

\section{Background and notation}

Symbols involving the letter $c$ denote constants used inside a single proof; their meaning does not persist beyond that. When a constant from one statement is to be used in the future, it will be denoted differently.

The symbol $\approx$ means equality up to a bounded multiplicative constant; bars $|\cdot|$ mean diameter when applied to sets.

A self-similar iterated function system (IFS) is a finite family $\left(f_{i}\right)_{i=1}^{k}$ of contracting similitudes from $\mathbb{R}^{d}$ to itself. Their contraction ratios are denoted by $\lambda_{i}$, the greatest of them by $\lambda^{+}$. The limit set of an IFS $\left(f_{i}\right)$ is the unique nonempty compact set $\Lambda$ satisfying

$$
\Lambda=\bigcup f_{i}(\Lambda)
$$

The symbolic space of an IFS is defined as $\Sigma=\{1, \ldots, k\}^{\mathbb{N}}$; its elements are denoted by $\omega=\left(\omega_{1} \omega_{2} \ldots\right)$. The truncation of $\omega$ after $n$ entries is denoted by $\omega^{n}=$ $\left(\omega_{1} \cdots \omega_{n}\right)$. We write $f_{\omega^{n}}=f_{\omega_{1}} \circ \cdots \circ f_{\omega_{n}}$ and denote by $\lambda_{\omega^{n}}$ the local contraction ratio of $f_{\omega^{n}}$. A right shift is a map $\sigma_{i}$ on $\Sigma$ given by $\sigma_{i}\left(\omega_{1} \omega_{2} \ldots\right)=\left(i \omega_{1} \omega_{2} \ldots\right)$, for some $i=1, \ldots, k$.

We define a projection from $\Sigma$ onto $\Lambda$ by

$$
\pi(\omega)=\lim _{n \rightarrow \infty} f_{\omega^{n}}(0) .
$$

When $x=\pi(\omega), \omega$ is called a symbolic expansion of $x$ (it need not to be uniquely defined). The dynamics on $\Lambda$ (given by the $f_{i}$ ) is a factor of the dynamics on $\Sigma$ given by right shifts $\sigma_{i}$ :

$$
f_{i} \circ \pi=\pi \circ \sigma_{i} .
$$

$U$ will denote a bounded, simply connected, open neighbourhood of $\Lambda$. A set of the form $U_{\omega^{n}}=f_{\omega^{n}}(U)$ is called a cylinder (in dynamic space).

We will also use the sets $\Sigma_{\omega^{n}}=\sigma_{\omega^{n}}(\Sigma)=\sigma_{\omega_{1}} \circ \cdots \circ \sigma_{\omega_{n}}(\Sigma)$, called cylinders (in symbolic space). It is easy to see that $\pi\left(\Sigma_{\omega^{n}}\right)=\Lambda_{\omega^{n}} \subset U_{\omega^{n}}$; the cylinders $U_{\omega^{n}}$ and $\Sigma_{\omega^{n}}$ are called dual.

We introduce a metric on $\Sigma$ given by $\rho(\omega, \tau)=\left|U_{\omega^{n}}\right|$, where $\omega^{n}=\tau^{n}$ but $\omega^{n+1} \neq \tau^{n+1}$. If $\omega_{1} \neq \tau_{1}$ then $\rho(\omega, \tau)=|U|$. This metric agrees with the product topology on $\Sigma$. In this metric $\pi$ is a Lipschitz mapping. 
A family of cylinders $\left\{U_{\omega^{n}}: \omega^{n} \in T\right\}$ is a Moran cover of size l and variation $c$ if the cylinders' diameters are between $c^{-1} l$ and $c l$, their dual cylinders are disjoint and they cover $\Sigma$. A Moran collection is a choice of Moran covers for all $l \leq|U|$, with uniformly bounded variation. We usually denote our Moran collections by $Z$, and each Moran cover by $Z_{l}$.

The similarity dimension of a self-similar IFS is the unique number $s$ satisfying

$$
\sum_{i} \lambda_{i}^{s}=1 \text {. }
$$

Let $\mu$ be the Bernoulli measure on $\Sigma$ defined by the probability vector $\left(\lambda_{1}^{s}, \ldots, \lambda_{k}^{s}\right)$. The natural measure of the IFS is the projection $v$ of $\mu$ under $\pi$; we have

$$
\mu\left(\Sigma_{\omega^{n}}\right)=\lambda_{\omega^{n}}^{s}=c\left|U_{\omega^{n}}\right|^{s} .
$$

Given a family of IFS's, a Moran cover for one parameter will not be in general a Moran cover for another one. However, we will assume that our mappings are contracting similitudes with contraction ratios of the form

$$
\lambda_{i}(t)=\lambda_{i}(\tilde{t})^{b(t)},
$$

for some $\tilde{t}$ and all $t$. Then a Moran cover for one $t$ is a Moran cover for all others, and the measure $\mu$ does not depend on $t$.

\section{Transversality}

We will consider not a single IFS but a $d$-dimensional family of them, each acting in $\mathbb{R}^{d}$. We will use $t=\left(t_{1}, \ldots, t_{d}\right)$ as a parameter and write the dependence on the parameter explicitly, whenever this matters; for example, the limit set will be written $\Lambda_{t}$. The parameter set $\bar{V}$ is assumed to be a topological closed ball in $\mathbb{R}^{d}$.

We assume the contractions $f_{i}(x ; t)$ are of class $C^{1+\beta}$ so long as $x$ remains in $U$; that is, the derivatives $\partial f_{i} / \partial t_{j}$ are $C^{\beta}$ with respect to both $x$ and $t$. The Hölder constant can be chosen universally.

We choose the bounded open simply connected set $U$ so that $U \supset \Lambda_{t}$ for all $t$. Fix for some $t$ a Moran collection Z. Because of (2-1), this is a Moran collection for other $t$, too, with variation bounded by a constant $L_{3}$ not depending on $t$. Cylinders dual to those in $Z_{l}$ have measure $\mu$ between $L_{4}^{-1} l^{s}$ and $L_{4} l^{s}$. Hence any $Z_{l}$ has between $L_{4}^{-1} l^{-s}$ and $L_{4} l^{-s}$ elements.

Lemma 3.1 [Rams 2002]. Given $\omega$, the projection $\pi_{t}(\omega)$ is $C^{1+\beta}$ with respect to $t$. The Hölder constant can be chosen independently of $\omega$.

Lemma 3.2 [Rams 2002]. Given $\tilde{t}$ and $\omega^{n}$, the function

$$
F_{x, \omega^{n}}(t)=\frac{\log \lambda_{\omega^{n}}(t)}{\log \lambda_{\omega^{n}}(\tilde{t})}
$$

is $C^{\beta}$, with Hölder constant independent of $\omega^{n}$. 
By $(2-1), s(t)=s(\tilde{t}) / b(t)$. Set $h_{\omega, \tau}(t)=\pi_{t}(\omega)-\pi_{t}(\tau)$.

The following definition was first introduced (in the one-dimensional situation) in [Pollicott and Simon 1995].

Definition 3.3. A family of iterated function systems satisfies the transversality condition at $u$ if there exists a constant $Y$ such that for any two words $\omega, \tau$ with $\omega_{1} \neq \tau_{1}$,

$$
\left|h_{\omega, \tau}(u)\right|<Y \Longrightarrow\left|\operatorname{det} D_{t} h_{\omega, \tau}\right|_{\mid t=u}>Y .
$$

Here $D_{t}$ is the Jacobi matrix with respect to $t$.

Lemma 3.4 [Rams 2002]. Assume the transversality condition is satisfied for an interval of parameters. For fixed $\omega$ and $\tau$, every component of $\left(h_{\omega, \tau}\right)^{-1}\left(B_{r}(0)\right)$, where $r<Y$, is contained in some ball of radius $L_{6} r, L_{6}$ not depending on $\omega$ or $\tau$.

Given two cylinders $U_{\omega^{n}}$ and $U_{\tau^{m}}$ we denote by $I\left(\omega^{n}, \tau^{m}\right)$ the set of parameter values for which $U_{\omega^{n}} \cap U_{\tau^{m}} \neq \varnothing$.

Note that when $l$ is very small $\left|U_{\omega^{n}}(t)\right|$ can be very different for different $t$. On the other hand, we have the following lemma stating that $\left|U_{\omega^{n}}(t)\right|$ does not vary too much for sufficiently close parameter values. (Note that the set of parameters for which the transversality condition is satisfied for a given family of iterated function systems is open.)

Lemma 3.5. Given $\varepsilon$, for $l$ small enough, $U_{\omega^{n}}(u) \in Z_{l}, t, u \in V$ and $|t-u| \leq$ $\left|U_{\omega^{n}}(t)\right|$,

$$
\left|\frac{\left|U_{\omega^{n}}(t)\right|}{\left|U_{\omega^{n}}(u)\right|}-1\right| \leq \varepsilon
$$

Proof. The function $\lambda_{\omega^{n}}(t)$ is no greater than $L_{3} l|U|^{-1}$. By Lemma 3.2,

$$
\left|\log f_{\omega^{n}}^{\prime}(x ; t)-\log f_{\omega^{n}}^{\prime}(x ; u)\right| \leq c l^{\beta} \log \left(c L_{3} \frac{l}{|U|}\right),
$$

and the right-hand side vanishes when $l$ goes to 0 . Thus, the derivative of the similitude $f_{\omega^{n}}^{-1}(\cdot ; t) \circ f_{\omega^{n}}(\cdot ; u)$ has determinant arbitrarily close to 1 for $n$ big enough. This mapping is a diffeomorphism between $U_{\omega^{n}}(t)$ and $U_{\omega^{n}}(u)$. We are done.

Proposition 3.6. Given $u$, if the family of IFS satisfies transversality at $u$, there exists a neighbourhood $W$ of $u$ and a constant $Z$ such that for any $l<|U|$ and all cylinder pairs $\left(U_{\omega^{n}}, U_{\tau^{m}}\right) \in Z_{l}(u) \times Z_{l}(u)$ with $\omega_{1} \neq \tau_{1}$, the set $W \cap I\left(\omega^{n}, \tau^{m}\right)$ can be covered with $K$ balls $B_{i}$ of diameter at most $K \min _{t \in B_{i}} \max \left(\left|U_{\omega^{n}}(t)\right|,\left|U_{\tau^{m}}(t)\right|\right)$.

Proof. We need only to prove the assertion for small $l$ because for big $l$ it is automatically satisfied. Take any $U_{\omega^{n}}, U_{\tau^{m}} \in Z_{l}(u)$, with $\omega_{1} \neq \tau_{1}$, and let $\omega, \tau$ be sequences in $\Sigma$ beginning with $\omega^{n}$ and $\tau^{m}$, respectively. Set $x(t)=\pi_{t}(\omega)$, $y(t)=\pi_{t}(\tau)$ and $H(t)=|x(t)-y(t)|$. 
Choose $\varepsilon>0$. The sets $U_{\omega^{n}}(u)$ and $U_{\tau^{m}}(u)$ have diameter approximately $l$. Hence, by Lemma 3.2, for $W$ small enough and $l$ small enough,

$$
\left|U_{\omega^{n}}(t)\right|<l^{1-\varepsilon} \quad \text { for any } t \in W,
$$

and the same for $U_{\tau^{m}}(t)$. Take $l$ so small that $l^{1-\varepsilon}<Y / 2$. We see that

$$
U_{\omega^{n}}(t) \subset B_{l^{1-\varepsilon}}(x(t)) \text { and } \quad U_{\tau^{m}}(t) \subset B_{l^{1-\varepsilon}}(y(t)) .
$$

When $t \in I\left(\omega^{n}, \tau^{m}\right)$, the points $x(t)$ and $y(t)$ have to be within distance at most $Y$ of each other. By Lemmas 3.4 and 3.5 transversality implies that any component of the set $\left\{t: H(t) \leq\left|U_{\omega^{n}}(t)\right|+\left|U_{\tau^{m}}(t)\right|\right\}$ is contained in a ball of radius no greater than $L_{6}(1+\varepsilon)\left(\left|U_{\omega^{n}}(\tilde{t})\right|+\left|U_{\tau^{m}}(\tilde{t})\right|\right)$, where $\tilde{t}$ is any parameter value belonging to this component, provided $l$ is small enough that we can use Lemma 3.5.

We have only to estimate the number of such components. Let $V_{1}$ and $V_{2}$ be any two of them. Draw a line segment $\alpha$ between the closest points of $V_{1}$ and $V_{2}$, and call these points $t^{(1)}$ and $t^{(2)}$. Then $H\left(t^{(i)}\right)=\left|U_{\omega^{n}}\left(t^{(i)}\right)\right|+\left|U_{\tau^{m}}\left(t^{(i)}\right)\right|$. By Lemma 3.4, $\left|H^{\prime}\left(t^{(i)}\right)\right| \geq c L_{6}^{-1}$, where the derivative is taken along $\alpha$. This derivative is positive for $i=1$ and negative for $i=2$. The function $H(t)=\left|h_{\omega, \tau}(t)\right|$ is (by Lemma 3.1) a $C^{1+\beta}$ function with bounded Hölder constant; hence its derivative cannot change too much in too short a distance. All in all, the length of $\alpha$ is bounded from below by a constant independent from $l, \omega, \tau, V_{1}, V_{2}$.

All the components of $\left\{t: H(t) \leq 2 l^{1-\varepsilon}\right\} \cap W$ are thus within a distance bounded from below from each other. Hence the number of them depends only on the size of $W$ and not on the size of the components. We are done.

\section{Intersection numbers}

Define

$$
\tilde{A}_{l}=\#\left\{\left(U_{\omega^{n}}, U_{\tau^{m}}\right) \in Z_{l} \times Z_{l}: U_{\omega^{n}} \cap U_{\tau^{m}} \neq \varnothing\right\}
$$

(note that we do not exclude the case $\omega^{n}=\tau^{m}$ ). We call this the intersection number. It lies between $\# Z_{l} \approx l^{-s}$ and $\#\left(Z_{l} \times Z_{l}\right) \approx l^{-2 s}$.

The geometric meaning of $\tilde{A}_{l}$ is given by the following proposition.

Proposition 4.1 [Rams 2002]. The limit

$$
\tilde{s}=\lim _{l \searrow 0} \frac{\log \tilde{A}_{l}}{-\log l}
$$

exists, and $2 s-\tilde{s}$ is equal to the correlation dimension of $v$. In particular, $\tilde{s}$ does not depend on the choice of $U$ and of the Moran collection.

It follows that if $s>d$ then $\tilde{A}_{l} \geq c l^{-2 s+d}$.

We define a second kind of intersection number:

$$
A_{l}=\#\left\{\left(U_{\omega^{n}}, U_{\tau^{m}}\right) \in Z_{l} \times Z_{l}: \omega_{1} \neq \tau_{1}, U_{\omega^{n}} \cap U_{\tau^{m}} \neq \varnothing\right\}
$$


To write explicitly the dependence of $A_{l}$ and $\tilde{A}_{l}$ we assume from now on that the Moran collection $Z$ is chosen in a special way. Namely, we demand that there exists a sequence $l_{i} \searrow 0$ such that every cylinder $U_{\omega^{n}}$ belongs to at least one and at most $L_{7}$ of the Moran covers $Z_{l_{i}}$. For $L_{7}$ big enough this is easy to satisfy. It is enough that such a sequence exists for one parameter value $u$; for other ones it follows from $(2-1)$, with $l_{i}(t)=l_{i}(u)^{b(t)}$.

Lemma 4.2 [Rams 2002]. There exists $\gamma<1$ and L such that for any $j>i$

$$
l_{j} \leq L \gamma^{j-i} l_{i}
$$

\section{Proposition 4.3.}

$$
L_{8}^{-1} l^{-s}\left(1+\sum_{l_{i} \geq L_{9}^{-1} l} l_{i}^{s} A_{l_{i}}\right) \leq \tilde{A}_{l} \leq L_{8} l^{-s}\left(1+\sum_{l_{i} \geq L_{9} l} l_{i}^{s} A_{l_{i}}\right) .
$$

This is the key fact. Now we can use transversality to get results about $A_{l}$ and translate them to results about $\tilde{A}_{l}$ (with important geometric consequences).

Lemma 4.4. Let $h$ be a positive Lipschitz function on $\mathbb{R}^{d}$ with Lipschitz constant not greater than $\frac{1}{4}$. Let $b$ be a positive integer function on $\mathbb{R}^{d}$. Let $\left(E_{i}\right)_{i=1}^{a} \subset \mathbb{R}^{d}$ be a family of sets such that $E_{i} \subset B_{h\left(x_{i}\right)}\left(x_{i}\right)$. Then the set $E^{\prime}$ of points $x \in \mathbb{R}^{d}$ belonging to at least $b(x)$ of the sets $E_{i}$ can be covered with a family of balls $F_{i}=B_{3 h\left(y_{i}\right)}\left(y_{i}\right)$ such that $\sum b\left(y_{i}\right) \leq a$.

Proof. Given $i$, we have $\frac{3}{4} h\left(x_{i}\right) \leq h(x) \leq \frac{5}{4} h\left(x_{i}\right)$ on $E_{i}$. Let $y_{1}$ be a point belonging to at least $b\left(y_{1}\right)$ of the sets $E_{i}$. For all of them we have $h\left(x_{i}\right)<\frac{4}{3} h\left(z_{1}\right)$. Hence the ball $F_{1}=B_{3 h\left(y_{1}\right)}\left(y_{1}\right)$ will contain all these $E_{i}$, because $2 \cdot \frac{4}{3}<3$.

Now choose any $y_{2} \notin F_{1}$ belonging to at least $b\left(y_{2}\right)$ of the sets $E_{i}$ - necessarily these will be different $i$ 's than for $y_{1}$ - and all these sets will be contained in $F_{2}$. We proceed in the same manner. The procedure will eventually stop because it decreases the number of sets $E_{i}$ at every step by at least $b\left(y_{i}\right)$. Hence $a \leq \sum b\left(y_{i}\right)$.

Theorem 4.5. Assume a d-parameter family of self-similar IFS satisfies (2-1) and transversality. Then for almost all parameter values $t$ there exist a positive $M$ and a sequence $z_{i} \searrow 0$ such that

$$
\begin{aligned}
& \text { - if } s(t)<d, \tilde{A}_{z_{i}}(t) \leq M z_{i}^{-s(t)}, \\
& \text { - if } s(t)>d, \tilde{A}_{z_{i}}(t) \leq M z_{i}^{-2 s(t)+d}, \\
& \text { - if } s(t)=d, \tilde{A}_{z_{i}}(t) \leq-M z_{i}^{-s(t)} \log z_{i} .
\end{aligned}
$$

Proof. It suffices to prove the assertion in a small neighbourhood of some parameter value $u$. We choose a neighbourhood $W$ such that the assertion of Proposition 3.6 at $u$ is satisfied.

We will use the notation $l(t)=l^{b(t)}$. Note that $l(t)^{s(t)}$ does not depend on $t$. 
By definition,

$$
\begin{aligned}
& \tilde{A}_{l}(t)=\sum_{U_{\omega^{n}} \in Z_{l}} \sum_{U_{\tau^{m} \in Z_{l}}} \mathbb{1}_{I\left(\omega^{n}, \tau^{m}\right)}(t), \\
& A_{l}(t)=\sum_{U_{\omega^{n} \in Z_{l}}} \sum_{\substack{U_{\tau^{m} \in Z_{l}} \\
\tau_{1} \neq \omega_{1}}} \mathbb{1}_{I\left(\omega^{n}, \tau^{m}\right)}(t),
\end{aligned}
$$

where $\mathbb{1}_{X}$ is the characteristic function of $X$.

We denote by $E(\phi(t))$ the average of $\phi$ with respect to the normalized Lebesgue measure on $W$ :

$$
E(\phi)=\frac{1}{m(W)} \int_{W} \phi(t) d m(t) .
$$

There are three cases: $s(u)<d, s(u)>d$ and $s(u)=d$.

Case I. From Proposition Proposition 3.6 we get for $U_{\omega^{n}}, U_{\tau^{m}} \in Z_{l}(u)$ and $\omega_{1} \neq \tau_{1}$

$$
E\left(l(t)^{-d} \cdot \mathbb{1}_{I\left(\omega^{n}, \tau^{m}\right)}\right) \leq K \cdot K^{d} .
$$

We claim that there exists a constant $M_{0}$ such

$$
E\left(l(t)^{s(t)} \tilde{A}_{l(t)}(t)\right) \leq M_{0} \quad \text { for all } l .
$$

If this is true, one easily checks that for any $l$ the set $\left\{t \in W: \tilde{A}_{l(t)}(t)>M\right\}$ has Lebesgue measure no greater than $\left(M_{0} / M\right) m(W)$ (this is a kind of Markov inequality). Hence the set $\left\{t \in W: \lim \inf \tilde{A}_{l(t)}(t)>M\right\}$ also has Lebesgue measure no greater than $\left(M_{0} / M\right) m(V)$ and the assertion follows.

To prove the claim, we may freely assume that $W$ is so small that for any parameter value $t \in V$ we have

$$
l(t)^{d}<l^{(1+\varepsilon) s} .
$$

Then

$$
E\left(l^{s} A_{l(t)}(t)\right)=l^{2 s} E\left(l^{-s} A_{l(t)}(t)\right) \leq l^{(2+\varepsilon) s} E\left(l(t)^{-d} A_{l(t)}(t)\right) .
$$

The last average is a sum of no more than $L_{4}^{2} l^{-2 s}$ averages of the form

$$
E\left(l(t)^{-d} 1_{I\left(\omega^{n}, \tau^{m}\right)}\right),
$$

which were estimated in (4-1). Hence

$$
E\left(l^{s} A_{l(t)}(t)\right) \leq K^{d+1} L_{4}^{2} l^{s \varepsilon} .
$$

Now we use second inequality in Proposition 4.3 and average both sides. We get

$$
E\left(l^{s} \tilde{A}_{l(t)}(t)\right) \leq L_{8}\left(1+\sum_{l_{i} \geq L_{9} l} E\left(l_{i}^{s} A_{l_{i}(t)}(t)\right)\right)
$$

and the sum on the right is part of an exponentially converging series. The whole right-hand side of this inequality is then bounded by a constant and (4-2) follows. 
Case II. Let

$$
G=\bigcap_{M} \bigcup_{l} \bigcap_{\tilde{l}<l}\left\{t \in W: \tilde{A}_{\tilde{l}} \geq M \tilde{l}^{d-2 s(t)}\right\}
$$

be the set of parameters for which the assertion of the theorem fails. We will first prove that

$$
G \subset \bigcap_{M} \bigcup_{l} \bigcap_{\tilde{l}<l} \bigcup_{l_{i}>\tilde{l}} G_{M, \tilde{l}, i}
$$

where

$$
G_{M, \tilde{l}, i}=\left\{t \in W: A_{l_{1}}(t)>M l_{i}^{\frac{1}{2} d-\frac{3}{2} s(t)} \tilde{l}(t)^{\frac{1}{2} d-\frac{1}{2} s(t)}\right\} .
$$

It is almost immediate. If for given $M$ and $\tilde{l}$ for all $i$ the parameter value $t \in W$ does not belong to $G_{M, \tilde{l}, i}$, Proposition 4.3 yields

$$
\begin{aligned}
\tilde{A}_{\tilde{l}(t)}(t) \tilde{l}(t)^{2 s(t)-d} & \leq L_{8} \tilde{l}(t)^{s(t)-d}\left(1+\sum_{l_{1} \geq L_{9} \tilde{l}} l_{i}(t)^{s(t)} A_{l_{i}(t)}(t)\right) \\
& \leq L_{8}+L_{8} M \cdot \sum_{i}\left(\frac{\tilde{l}(t)}{l_{i}(t)}\right)^{\frac{1}{2}(s(t)-d)} \leq c M,
\end{aligned}
$$

and the contradiction proves (4-5).

Now we estimate the size of $G$. By Proposition 3.6, the union of the sets $I\left(\omega^{n}, \tau^{m}\right)$ over all $U_{\omega^{n}}, U_{\tau^{m}} \in Z_{l_{i}}(u)$ can be covered (with multiplicities) by at most $a=L_{4}^{2} K l_{i}^{-2 s(u)}=L_{4}^{2} K l_{i}(t)^{-2 s(t)}$ sets $E_{i}$ satisfying

$$
\left|E_{i}\right| \leq K \max \left\{\left|U_{\omega^{n}}(t)\right|: U_{\omega^{n}}(u) \in Z_{l_{i}}(u)\right\}=: h(t) \approx l_{i}(t),
$$

where $t$ is any point from $E_{i}$.

The set $G_{M, \tilde{l}, i}$ is the set of points $t$ that belong to at least

$$
b(t)=M l_{i}(t)^{\frac{1}{2} d-\frac{3}{2} s(t)} \tilde{l}(t)^{\frac{1}{2} d-\frac{1}{2} s(t)}
$$

of the sets $E_{i}$. By Lemma $4.4, G_{M, \tilde{l}, i}$ can be covered by a family of balls $F_{i}=$ $B_{3 h\left(y_{i}\right)}\left(y_{i}\right)$ such that

$$
\sum_{j} M l_{i}\left(y_{j}\right)^{\frac{1}{2} d-\frac{3}{2} s\left(y_{j}\right)} \tilde{l}\left(y_{j}\right)^{\frac{1}{2} d-\frac{1}{2} s\left(y_{j}\right)} \leq L_{4}^{2} K l_{i}(u)^{-2 s(u)} .
$$

The right-hand side of this inequality will not change if we replace $u$ by any $y_{j}$. Hence,

$$
2^{d} \cdot \sum_{j} l_{i}\left(y_{j}\right)^{d} \leq 2^{d} L_{4}^{2} K M^{-1} \sup _{t \in W}\left(\frac{\tilde{l}(t)}{l_{i}(t)}\right)^{\frac{1}{2} s(t)-\frac{1}{2} d} .
$$


The left-hand side of this inequality is an upper bound for the $d$-dimensional volume of the union of all the balls $F_{i}$, hence also for $G_{M, \tilde{l}, i}$. Hence,

$$
\operatorname{vol}\left(\bigcup_{l_{i}>\tilde{l}} G_{M, \tilde{l}, i}\right) \leq 2^{d} L_{4}^{2} K M^{-1} \sum_{l_{i}>\tilde{l}} \sup _{t \in W}\left(\frac{\tilde{l}(t)}{l_{i}(t)}\right)^{\frac{1}{2} s(t)-\frac{1}{2} d} .
$$

Since $W$ may be chosen small enough that $s(t) \geq \frac{1}{2}(s(u)+d)$ for all $t \in W$, the sum can be bounded from above by a constant independent from $\tilde{l}$. Hence

$$
\operatorname{vol}\left(\bigcap_{\tilde{l}<l} \bigcup_{l_{i}>\tilde{l}} G_{M, \tilde{l}, i}\right) \leq c M^{-1}
$$

As $l$ tends to 0 , the sets such as the above one form an increasing sequence, so the measure of their union is equal to the limit of their measures, hence it is also no greater than $c M^{-1}$. Letting $M$ go to infinity we get the assertion.

Case III. We assume that $s(t)=d$ for a set of parameters of positive Lebesgue measure, otherwise the assertion is empty. We repeat the proof of case I with very small changes. Instead of averaging over all of $W$, we average over $W_{0}=W \cap\{t$ : $s(t)=d\}$. Now (4-1) takes the form

$$
E\left(l(t)^{-d} \mathbb{1}_{I\left(\omega^{n}, \tau^{m}\right)}\right) \leq K \cdot K^{d} \frac{m(W)}{m\left(W_{0}\right)} .
$$

Instead of (4-2), we have to prove

$$
E\left(l(t)^{s(t)} \tilde{A}_{l(t)}(t)\right)<-M_{0} \log l .
$$

We estimate the left-hand side of this formula in the same way as before, using $s(t)=d$ instead of (4-3). In place of (4-4) we will get

$$
E\left(l^{s} A_{l(t)}(t)\right)=l^{2 s} E\left(l^{-s} A_{l(t)}(t)\right) \leq l^{2 s} E\left(l(t)^{-d} A_{l(t)}(t)\right) \leq L_{4}^{2} K^{d+1} \frac{m(W)}{m\left(W_{0}\right)} .
$$

Substituting this into Proposition 4.3, we conclude that $E\left(l^{s} \tilde{A}_{l(t)}(t)\right)$ is no greater than a sum of bounded summands, taken over all $l_{i} \geq l$. By Lemma 4.2, this sum has approximately $-\log l$ elements, and the assertion follows.

\section{Geometric consequences of the bounds on intersection numbers}

The following lemma is an immediate consequence of [Rams 2002, Lemma 5.6].

Lemma 5.1. Among the cylinders $U_{\omega^{n}} \in Z_{l}$ one can choose a pairwise disjoint family having at least $\mathrm{cl}^{-2 s} / \tilde{A}_{l}$ elements.

Hence, for a typical parameter $t$ such that $s(t)<d$, Theorem 4.5 implies that for many scales $l$ some percentage (depending on $t$ but not on $l$ ) of cylinders from $Z_{l}$ is pairwise disjoint. For $s(t)>d$ we get as many disjoint cylinders as possible, too. For $s(t)=d$ we cannot hope to get the same kind of estimate because this would 
imply positivity of the Lebesgue measure of the limit set, and hence, by [Schief 1994], the open set condition.

We will work with the natural measure $v$. We approximate $v$ by a sequence of measures $v_{l}$, absolute continuous with respect to the $d$-dimensional Lebesgue measure and with density

$$
D_{v_{l}}=\frac{1}{\sum_{U_{\omega^{n}} \in Z_{l}} m\left(U_{\omega^{n}}\right)} \sum_{U_{\omega^{n}} \in Z_{l}} \mathbb{1}_{U_{\omega^{n}}} .
$$

As the Moran cover $Z_{l}$ has approximately $l^{-s}$ cylinders, each of volume $\approx l^{d}$, the first fraction approximately equals $l^{s-d}$.

We treat the situation $s(t)>d$ first. Recall from [Mattila 1995, pp. 36 and 43], for example, that $v$ is absolutely continuous with respect to the Lebesgue measure with $L^{2}$ density if and only if

$$
\int \underline{D}_{v} d v<\infty
$$

where $\underline{D}$ stands for lower density with respect to $d$-dimensional Lebesgue measure.

Lemma 5.2. $\underline{D}_{v} \approx \liminf _{l \searrow 0} D_{v_{l}}$.

Proof. At points outside $\Lambda$, both sides of the formula are zero. We thus need to check it only for points from $\Lambda$. Choose any $x \in \Lambda$. We estimate the measure of a ball $B_{r}(x)$ from above and from below: for all $l$ we have

$$
v\left(B_{r}(x)\right) \leq L_{4} l^{s} \#\left\{U_{\omega^{n}} \in Z_{l}: B_{r}(x) \cap \pi\left(\Sigma_{\omega^{n}}\right) \neq \varnothing\right\}
$$

and

$$
v\left(B_{r}(x)\right) \geq L_{4}^{-1} l^{s} \#\left\{U_{\omega^{n}} \in Z_{l}: U_{\omega^{n}} \subset B_{r}(x)\right\} .
$$

There exist $L_{1}, L_{2}$ such that $B_{L_{1}|U|}(\Lambda) \subset U \subset B_{L_{2}|U|}(\Lambda)$. The pair $(U, \Lambda)$ is mapped by any $f_{\omega^{n}}$ in a self-similar way, hence

$$
B_{L_{1} \lambda_{\omega^{n}}}\left(\pi\left(\Sigma_{\omega^{n}}\right)\right) \subset U_{\omega^{n}} \subset B_{L_{2} \lambda_{\omega^{n}}}\left(\pi\left(\Sigma_{\omega^{n}}\right)\right)
$$

for all $\omega^{n}$.

Hence, given any $x \in \Lambda$, any $l \leq|U|$ and any $U_{\omega^{n}} \in Z_{l}$ and $x \in \pi\left(\Sigma_{\omega^{n}}\right)$, we have $B_{L_{1} L_{3}^{-1} l}(x) \subset U_{\omega^{n}}$ (because of the uniform variation of the Moran collection). For the same reason the ball $B_{\left(L_{2}+1\right) L_{3} l}(x)$ contains all the cylinders from $Z_{l}$ intersecting $x$. Hence

$$
B_{r}(y) \cap \pi\left(\Sigma_{\omega^{n}}\right) \neq \varnothing \Longrightarrow y \in U_{\omega^{n}}
$$

when $U_{\omega^{n}} \in Z_{L_{1}^{-1} L_{3} r}$. Since

$$
D_{v_{l}}(y)=l^{s-d} \#\left\{U_{\omega^{n}} \in Z_{l}: y \in U_{\omega^{n}}\right\}
$$


we get from $(5-1)$

$$
\frac{v\left(B_{r}(y)\right)}{r^{d}} \leq L_{4}\left(L_{1}\right)^{s-d} D_{v_{L_{1}^{-1} r}}(y) .
$$

Estimations in the other direction use the other property: if $x \in U_{\omega^{n}} \in Z_{l}$ then $U_{\omega^{n}} \subset B_{L_{2} l}(x)$. Together with (5-2) this gives

$$
\frac{v\left(B_{r}(x)\right)}{r^{d}} \geq L_{4}^{-1}\left(\left(L_{2}+1\right) L_{3}\right)^{d} D_{v_{\left(L_{2}+1\right)}-1 L_{3}^{-1} r} .
$$

The lemma follows from (5-3) and (5-4) by passing to a (lower) limit with $r$ going to 0 .

Now we get the condition written in terms of intersection numbers and their growth.

Proposition 5.3. If $\int \underline{D}_{v} d v=\infty$ then $\lim _{l \searrow 0} l^{2 s-d} \tilde{A}_{l}=\infty$.

Proof. We will prove this proposition under an additional assumption on the shape of $U$. Namely, we demand that

$$
m\left(U_{\omega^{n}} \cap B_{r}(x)\right) \geq c r^{d},
$$

provided $x \in U_{\omega^{n}}$ and $r \leq\left|U_{\omega^{n}}\right|$. We can do this because the assumption of the proposition does not depend at all on the choice of $U$ and the function under limit in the assertion will change only by a constant (so the limit will not change). This assumption we add is void when $d=1$ (with $c=\frac{1}{2}$ ); for greater $d$ we need to check it only for $U$.

We claim first that

$$
\int D_{v_{l}} d v \leq c_{0}^{-1} \int D_{v_{l}} d v_{l^{\prime}}
$$

for some constant $c_{0}$ for all $l^{\prime} \leq l$.

Assuming that, it is easy to get the assertion. From Fatou's lemma we get

$$
\liminf _{l \searrow 0} \int D_{v_{l}} d v_{l} \geq c_{0} \liminf _{l \searrow 0} \int D_{v_{l}} d v \geq c_{0} \int \liminf _{l \searrow 0} D_{v_{l}} d v \approx \int \underline{D}_{v} d v .
$$

We may use Fatou's lemma here because any weak sublimit of the measures $v_{l}$ (with $l$ going down to 0 ) is equivalent to $v$, with density bounded from above and below.

The left-hand side of this chain of inequalities is easy to write using intersection numbers:

$$
\int D_{v_{l}} d v_{l} \approx l^{2(s-d)} \sum_{U_{\omega^{n} \in Z_{l}}} \sum_{U_{\tau^{m}} \in Z_{l}} m\left(U_{\omega^{n}} \cap U_{\tau^{m}}\right) \leq\left(2 L_{3}\right)^{d} l^{2 s-d} \tilde{A}_{l},
$$

and we are done.

Now we prove (5-6). Take any $U_{\omega^{n}} \in Z_{l}$. If it contains any point $x \in \pi\left(\Sigma_{\tau^{m}}\right) \subset$ $U_{\tau^{m}} \in Z_{l^{\prime}}$, the intersection $U_{\omega^{n}} \cap U_{\tau^{m}}$ contains a big part of the ball $B_{L_{1} L_{3}^{-1} l^{\prime}}(x)$, as 
in the proof of Lemma 5.2. This is so because this ball is fully contained in $U_{\tau^{m}}$ and its intersection with $U_{\omega^{n}}$ is of volume at least $c\left(L_{1} L_{3}^{-1} l^{\prime}\right)^{d}$, by (5-5). Hence

$$
\int \mathbb{1}_{U_{\omega^{n}}} d v_{l^{\prime}} \geq c\left(L_{1} L_{3}^{-1} l^{\prime}\right)^{d}\left(l^{\prime}\right)^{s-d} \#\left\{U_{\tau^{m}} \in Z_{l^{\prime}}: \pi\left(\Sigma_{\tau^{m}}\right) \cap U_{\omega^{n}} \neq \varnothing\right\} .
$$

But we can write also

$$
\int \mathbb{1}_{U_{\omega^{n}}} d v \leq L_{4}\left(l^{\prime}\right)^{s} \#\left\{U_{\tau^{m}} \in Z_{l^{\prime}}: \pi\left(\Sigma_{\tau^{m}}\right) \cap U_{\omega^{n}} \neq \varnothing\right\} .
$$

As the density of $v_{l}$ is just a sum of characteristic functions of cylinders from $Z_{l}$ multiplied by a positive constant, our claim follows with $c_{0}=L_{4}^{-1} L_{1}^{d} L_{3}^{-d} c$.

This proposition together with Theorem 4.5 gives us the following result, first proved (in less generality) by Solomyak [1995] and then by Peres and Schlag [2000]:

Theorem 5.4. Under the assumptions of Theorem 4.5, for almost every parameter value $t$ such that $s(t)>d$ the natural measure $v$ is absolutely continuous with respect to the Lebesgue measure and its density is in $L^{2}$.

Now we work on the case $s(t) \leq d$. We introduce a special notation: $x^{s^{*}}$ will denote either $x^{s}$ (when $s<d$ ) or $-x^{s} \log x$ (when $s=d$ ). By the lower $s^{*}$-density of a measure we will mean

$$
\underline{D}_{s}(v, x)=\liminf _{r \rightarrow 0} \frac{v\left(B_{r}(x)\right.}{r^{s^{*}}} .
$$

By [Taylor and Tricot 1985, Theorem 5.4], if $\underline{D}_{s^{*}}(v, x)$ is finite for $v$-almost all $x$ then the packing measure of the limit set (with gauge function $x^{s^{*}}$ ) is positive and $v$ is absolutely continuous with respect to this packing measure restricted to the limit set.

Proposition 5.5. If $\liminf l^{s^{*}} \tilde{A}_{l} \leq M_{0}$ then $v\left\{x \in \Lambda: \underline{D}_{s^{*}}(v, x)>M\right\} \leq c M_{0} / M$.

Proof. The proof is similar to one of Lemma 5.2 in the case $s>d$. We will use the measures $v_{l}$ as we did there, and get

$$
\underline{D}_{s^{*}}(v, x) \approx \liminf _{l \rightarrow 0} \frac{v_{l}\left(B_{c^{\prime} l}(x)\right)}{\left(c^{\prime} l\right)^{s^{*}}} .
$$

Then we may estimate $v_{l}\left(B_{c^{\prime} l}(x)\right)$ by $L_{4} l^{s}$ times the number of cylinders from $Z_{l}$ that do intersect $\left.B_{c^{\prime} l}(x)\right)$. For all $x \in \pi\left(\Sigma_{\omega^{n}}\right)$, this number is no greater than the number - call it $N_{l}\left(U_{\omega^{n}}\right)$ - of cylinders from $Z_{l}$ that intersect $U_{\omega^{n}}$. When we sum up $N_{l}\left(U_{\omega^{n}}\right)$ for all $U_{\omega^{n}} \in Z_{l}$, we will get $\tilde{A}_{l}$.

We choose an $l$ such that $\tilde{A}_{l} \leq 2 M_{0} l^{s^{*}}$ (by assumption, we can find such an $l$ as small as we wish). Then $N_{l}\left(U_{\omega^{n}}\right)$ is greater than $M$ (when $s<d$ ) or $-M \log l$ (when $s=d$ ) for not more than $2 L_{3} M_{0} l^{-s} / M$ cylinders from $Z_{l}$ (there are between 
$L_{3}^{-1} l^{-s}$ and $L_{3} l^{-s}$ cylinders in $\left.Z_{l}\right)$. Hence, for all the points from $\pi\left(\Sigma_{\tau^{m}}\right)$ where $U_{\tau^{m}}$ is not one of these cylinders, we have

$$
\frac{\nu_{l}\left(B_{c^{\prime} l}(x)\right)}{\left(c^{\prime} l\right)^{s^{*}}}<c^{\prime \prime} M .
$$

The set of these points - call it $G_{l}$ - has measure $v$ no smaller than $1-c M / M_{0}$.

Now we pass to the limit with $l$ going to 0 . All points from the set $\lim \sup G_{l}$ have lower $s^{*}$-density no greater than $c^{\prime \prime} M$, and $v\left(\lim \sup G_{l}\right) \geq 1-c M / M_{0}$. We are done.

The following theorem, whose first part was proved in [Peres et al. 2000], is an immediate consequence.

Theorem 5.6. Under the assumptions of Theorem 4.5, for almost all parameters $t$ with $s(t) \leq d$,

- if $s(t)<d$, the $s$-dimensional packing measure of $\Lambda$ is positive and the measure $v$ is absolutely continuous with respect to the packing measure;

- if $s(t)=d$, the packing measure with gauge function $\phi(x)=-x^{d} \log x$ of $\Lambda$ is positive and the measure $v$ is absolutely continuous with respect to this measure.

\section{References}

[Hutchinson 1981] J. E. Hutchinson, "Fractals and self-similarity", Indiana Univ. Math. J. 30:5 (1981), 713-747. MR 82h:49026 Zbl 0598.28011

[Mattila 1995] P. Mattila, Geometry of sets and measures in Euclidean spaces: fractals and rectifiability, Cambridge Studies in Advanced Mathematics 44, Cambridge University Press, Cambridge, 1995. MR 96h:28006 Zbl 0819.28004

[Peres and Schlag 2000] Y. Peres and W. Schlag, "Smoothness of projections, Bernoulli convolutions, and the dimension of exceptions", Duke Math. J. 102:2 (2000), 193-251. MR 2001d:42013 Zbl 0961.42007

[Peres et al. 2000] Y. Peres, K. Simon, and B. Solomyak, "Self-similar sets of zero Hausdorff measure and positive packing measure", Israel J. Math. 117 (2000), 353-379. MR 2001g:28017 Zbl 0963.28008

[Pollicott and Simon 1995] M. Pollicott and K. Simon, "The Hausdorff dimension of $\lambda$-expansions with deleted digits", Transactions of the Amer. Math. Soc. 347:3 (1995), 967-983. MR 95h:11080 Zbl 0831.28005

[Rams 2002] M. Rams, "Packing dimension estimation for exceptional parameters", Israel J. Math. 130 (2002), 125-144. MR 2003e:28028 Zbl 1005.37008

[Schief 1994] A. Schief, "Separation properties for self-similar sets", Proc. Amer. Math. Soc. 122:1 (1994), 111-115. MR 94k:28012 Zbl 0807.28005

[Solomyak 1995] B. Solomyak, "On the random series $\sum \pm \lambda^{n}$ (an Erdős problem)", Ann. of Math.

(2) 142:3 (1995), 611-625. MR 97d:11125 Zbl 0837.28007

[Taylor and Tricot 1985] S. J. Taylor and C. Tricot, "Packing measure, and its evaluation for a Brownian path”, Trans. Amer. Math. Soc. 288:2 (1985), 679-699. MR 87a:28002 Zbl 0537.28003 
Received April 26, 2002.

Michą RAMS

InSTITUTE OF MATHEMATICS

Polish ACADEMY OF SCIENCES

UL. ŚNIADECKICH 8, 00-950 WARSZAWA

POLAND

rams@impan.gov.pl 